\title{
THE LONG-LIVED UV “PLATEAU” OF SN 2012aw
}

\author{
Amanda J. Bayless ${ }^{1}$, Tyler A. Pritchard ${ }^{2}$, Peter W. A. Roming ${ }^{1,2}$, Paul Kuin ${ }^{3}$, Peter J. Brown ${ }^{4}$, \\ Maria Teresa Botticella ${ }^{5}$, Massimo Dall'Ora ${ }^{5}$, Lucille H. Frey ${ }^{6,7}$, Wesley Even $^{6}$, Chris L. Fryer ${ }^{6,8,9}$, \\ Justyn R. MAund ${ }^{10,11,12}$, AND Morgan Fraser ${ }^{10}$ \\ ${ }^{1}$ Southwest Research Institute, Department of Space Science, 6220 Culebra Road, San Antonio, TX 78238, USA \\ 2 Department of Astronomy \& Astrophysics, Penn State University, 525 Davey Lab, University Park, PA 16802, USA \\ ${ }^{3}$ Mullard Space Science Laboratory, Holmbury St. Mary, Dorking, Surrey RH5 6NT, UK \\ ${ }^{4}$ Texas A\&M University, Department of Physics and Astronomy, College Station, TX 77843-4242, USA \\ ${ }^{5}$ INAF-Osservatorio Astronomico di Capodimonte, via Moiariello 16, I-80131 Napoli, Italy \\ ${ }^{6}$ Los Alamos National Laboratory, Los Alamos, NM 87545, USA \\ ${ }^{7}$ Department of Computer Science, University of New Mexico, Albuquerque, NM 87131, USA \\ ${ }^{8}$ Physics Department, University of Arizona, Tucson, AZ 85721, USA \\ ${ }^{9}$ Physics and Astronomy Department, University of New Mexico, Albuquerque, NM 87131, USA \\ ${ }^{10}$ Astrophysics Research Center, School of Mathematics and Physics, Queen's University Belfast, Belfast BT7 1NN, UK \\ ${ }^{11}$ Dark Cosmology Centre, Niels Bohr Institute, University of Copenhagen, Juliane Maries Vej, DK-2100 Copenhagen, Denmark \\ Received 2012 October 19; accepted 2013 January 16; published 2013 January 29
}

\begin{abstract}
Observations with the Swift UV Optical Telescope have unambiguously uncovered for the first time a long-lived, UV "plateau" in a Type II-P supernova (SN). Although this flattening in slope is hinted at in a few other SNe, due to its proximity and minimal line-of-sight extinction, SN 2012aw has afforded the first opportunity to clearly observe this UV plateau. The observations of SN 2012aw revealed all Swift UV and $u$-band light curves initially declined rapidly, but 27 days after the explosion the light curves flattened. Some possible sources of the UV plateau are the same thermal process that causes the optical plateau, heating from radioactive decay, or a combination of both processes.
\end{abstract}

Key words: supernovae: general - supernovae: individual (SN 2012aw)

Online-only material: color figures

\section{INTRODUCTION}

A Type II-P supernova ( $\mathrm{SN}$ ) is the most common type of $\mathrm{SNe}$ in a volume-limited sample ( $\mathrm{Li}$ et al. 2011 and references therein) and results from the core collapse of a massive star, usually a red supergiant (RSG). The gravitational binding energy is approximately $10^{53} \mathrm{erg}$, but only a small percentage of this is eventually released as sustained emission with a lifetime of a few months. The luminosity in the optical is maintained by the recombination of hydrogen and excitation by radioactive isotopes even as the expanding photosphere cools. The initial emission is very blue, with the peak of the bolometric luminosity in the UV, but as the photosphere expands and cools, it becomes redder (Kirshner et al. 1973; Kirshner \& Kwan 1975; Mitchell et al. 2002; Leonard et al. 2002; Brown et al. 2007). Thus, the optical light curve often remains at nearly constant brightness (plateaus) for several weeks.

In spite of the copious number of Type II-P SNe, very few have been well studied in the UV since this requires space-based instruments and a nearby event with low extinction. Recent UV observations have been carried out by the Swift (Gehrels et al. 2004) UV Optical Telescope (UVOT; Roming et al. 2000, 2004, 2005), IUE (SN 1987A; Pun et al. 1995), the Hubble Space Telescope (HST; SN 1999em; Baron et al. 2000), and GALEX (SN 2005ay; Gal-Yam et al. 2008). These SNe are UV bright early on and can be seen at great distances, but the UV flux usually fades after a few weeks as the photosphere cools (Brown et al. 2009). For most distant SNe this means that the late-time UV flux is too faint to be observable. In the rare cases where the $\mathrm{SNe}$ were visible in the UV at late epochs, there are indications

\footnotetext{
${ }^{12}$ Royal Society Research Fellow.
}

of a change to a flatter slope, but there are little data at these late times to determine anything definitive. ${ }^{13}$ This has changed with the advent of SN 2012aw.

SN 2012aw was discovered on 2012 March 16.9 (Fagotti 2012) in the nearby galaxy M95 (10 Mpc; Freedman et al. 2001). M95 was observed on March 15.3 (Poznanski et al. 2012) without a detection to a limiting magnitude of $R>20.7$. From this constraint, Fraser et al. (2012) adopt an explosion date of 2012 March $16.0 \pm 0.8 \mathrm{~d}$ (JD 2456002.5), which we adopt as Day 0 throughout this Letter. Pre-explosion images of M95 in the region of SN 2012aw indicate an IR bright star in the vicinity, likely an RSG (Fraser et al. 2012; Van Dyk et al. 2012). Followup spectra (Munari 2012; Itoh et al. 2012; Siviero et al. 2012) identified it as a core collapse, with a preliminary classification as a Type II-P. The plateau classification is confirmed by the optical photometry from Swift as shown in this Letter. The nearness and minimal total extinction (line of sight: $A_{v}=0.37$, host: $A_{v}=0.24$, with $R_{v}=3.1$; Van Dyk et al. 2012) of SN 2012aw meant that unprecedented UV observations of an SN covering the first 111 days past explosion could be obtained. We also observed this SN with the UVOT UV grism for approximately two weeks post-explosion before it faded beyond a reasonable signal-to-noise threshold.

As in prior Type II-P SNe, SN 2012aw is initially UV bright followed by a rapid decline in flux. At about Day 27 the UV light curve slope significantly flattened to a very shallow sloped decline, a UV "plateau." This UV plateau continued though to Day 111, at which time Swift could no longer observe the SN due to observing constraints. In this Letter, we present the Swift optical and UV light curves. We also present and compare the

\footnotetext{
${ }^{13}$ SN 1987A was extensively observed, but it was also peculiar.
} 


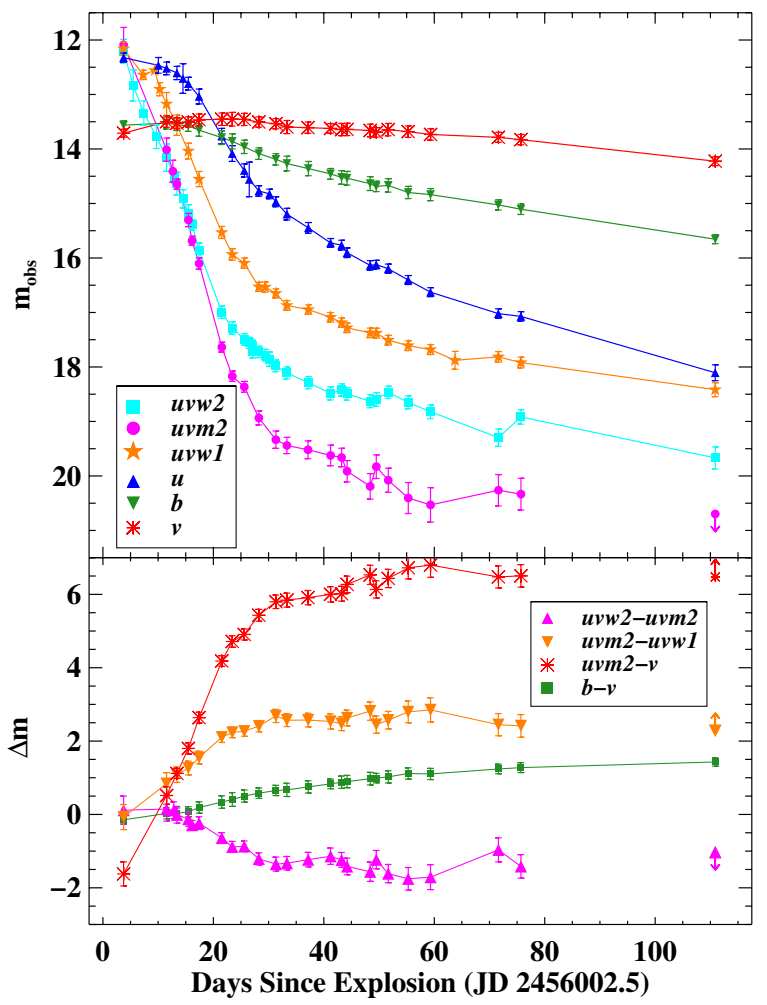

Figure 1. Top: the UV and optical light curves of SN 2012aw from Swift UVOT. The optical $v$ and $b$ bands are typical of a Type II-P SN. The UV bands decrease in brightness rapidly, but $\sim 27$ days past explosion the light curve flattens out and follows a similar slope as the optical bands. Bottom: UV and optical colors of SN 2012aw. The uvm $2-v$ (UV-optical) color curve indicates that the SN becomes quite red over time.

(A color version of this figure is available in the online journal.)

time-series UV spectra of SN 2012aw to previously observed spectral models of Type II-P SNe (SN 2005cs and SN 2006bp) and present possible causes of the UV flattening.

\section{OBSERVATIONS AND DATA REDUCTION}

\subsection{Swift UVOT Photometry}

SN 2012aw was observed from 2012 March 19 (UT; 3 days post-explosion) to May 30 (JD 2456006.2 to 2456078.2 ) with the Swift UVOT with a cadence of every one to four days. The UVOT observed in six UV/optical filters: $u v w 2\left(\lambda_{c}=1928 \AA\right)$, $u v m 2\left(\lambda_{c}=2246 \AA\right), u v w 1\left(\lambda_{c}=2600 \AA\right), u\left(\lambda_{c}=3465 \AA\right)$, $b\left(\lambda_{c}=4392 \AA\right)$, and $v\left(\lambda_{c}=5468 \AA\right.$; Poole et al. 2008). Photometry using a $3^{\prime \prime}$ source aperture, including galaxy flux subtraction using a previous Swift UVOT observation of M95 as a template, was performed following the method outlined in Brown et al. (2009). The data reduction pipeline used HEASOFT 6.6.3 and Swift Release 3.3 analysis tools with UVOT zero points (Poole et al. 2008) and updated calibrations (Breeveld et al. 2011).

The upper panel of Table 1 gives a summary of the photometric observations. Figure 1 shows the multi-color light curve and UV/optical colors of SN 2012aw. The $v$ and $b$ bands are largely flat, with declining slopes over the range of observations of $0.006 \pm 0.001$ and $0.023 \pm 0.001 \mathrm{mag}^{-1 a y}{ }^{-1}$, respectively ( $1 \sigma$ errors), the characterizing signature of a Type II-P SN. The SN is UV bright initially, but declines rapidly with slopes for the $u v w 2, u v m 2$, and $u v w 1$, of $0.311 \pm 0.012$, $0.197 \pm 0.010$, and $0.257 \pm 0.007 \mathrm{mag} \mathrm{day}^{-1}$, respectively.
After Day 27, the light curves flattened into a slowly declining plateau with slopes very similar to the $b$ band of $0.028 \pm 0.003$, $0.029 \pm 0.005$, and $0.031 \pm 0.002 \mathrm{mag} \mathrm{day}^{-1}$, respectively. The $u$-band light curve had two changes in slope. The initial trend was rather flat, similar to the optical light curves, with a slope of $0.038 \pm 0.007 \mathrm{mag} \mathrm{day}^{-1}$ until about Day 15 (JD 2456018). Then, the $u$ band followed the trend of the UV light curves, decreasing in brightness at a rate of $0.166 \pm 0.004$ mag day $^{-1}$ until about Day 27-30, where its light curve showed a slower decline of $0.050 \pm 0.003 \mathrm{mag} \mathrm{day}^{-1}$.

The $u v m 2-v$ color overall becomes quite red, as expected as the photosphere cools. The $u v w 2-u v m 2$ color does become blue initially. This is because the $u v m 2$ flux declines faster than the $u v w 2$ flux. Figure 2 shows the time-series UV spectra evolution, which is described in the following section. The flux near $1900 \AA$ $(u v w 2)$ remains nearly constant as the blackbody peak moves through this wave band and there are not many metal lines at this temperature $(\sim 20,000 \mathrm{~K}$; bolometric temperature, see Section 4). Near $2100 \AA$ (uvm2) in the first spectrum there is a flux peak from the absence of iron lines. In the later spectra, this is line blanketed most likely by Ni II. A similar phenomenon happened with SN 1987A (Cassatella et al. 1987).

\subsection{Swift UVOT Spectroscopy}

Swift UVOT started observing with the UV grism (calibrated for the range $\lambda 1650-\lambda 4900 \AA$ ) on 2012 March 21 (UT; $\sim 5$ days post-explosion). Spectra from individual images were summed in order to limit the noise in the spectrum (Figure 2). The bottom panel of Table 1 lists the start and end times of each summed observation and the total exposure time. The spectral features are redshifted due to M95's recessional velocity of $788 \mathrm{~km} \mathrm{~s}^{-1}$.

A feature of the Swift UV grism is the ability to observe at offsets from the center (default) position. These positions take advantage of the order separation, which is more pronounced when the grism is not at the default position. This means that the blue part of the spectrum can also be observed in second order, and the contamination of the first order by the second order is reduced substantially. All but the last spectrum were observed at offset positions. The offset was moved closer to the central position as time went on. A new preliminary calibration for the offset positions was used (P. Kuin et al., in preparation), which has an accuracy of $15 \AA$ and is reliable to within $20 \%$ below $1800 \AA$ and $<10 \%$ for the UV region between 1800 and $4900 \AA$. The spectra were extracted following the same procedure as for SN 2011ht (Roming et al. 2012), using version 0.9.6 of the Python software for UVOT grism analysis. ${ }^{14}$ The last spectrum was observed at the central default position because the UV emission was greatly reduced already, meaning that the blue part of the second order gives minimal contamination to the first order. The first five spectra have second-order contamination starting at $\sim 4900 \AA$ which is responsible for the upward jump in the flux. The last two spectra have some contamination by a zeroth order between 2030 and $2167 \AA$, which was removed, producing the gap in Figure 2. The background noise level in the spectra is $6 \times 10^{-16} \mathrm{erg} \mathrm{cm}^{-2} \mathrm{~s}^{-1} \AA^{-1}$. The last spectrum blueward of $2940 \AA$ is below this noise level.

To check the calibrations we produced spectrophotometric Swift magnitudes from the spectra in Figure 2, which are tabulated in Table 1 . The UV grism does not completely cover the $u v w 2$ and the $v$ band and therefore these are only limits. The UV magnitudes from the last spectrum are not included because

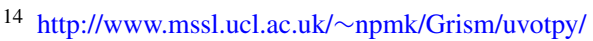


Table 1

Observations of SN 2012aw from UVOT

\begin{tabular}{|c|c|c|c|c|c|c|}
\hline $\mathrm{JD}(+2450000)$ & $u v w 2$ & $\begin{array}{l}\text { Photometric } \\
\text { uvm2 }\end{array}$ & $\begin{array}{c}\text { Magnitudes }^{\mathrm{a}} \\
\text { uvw1 }\end{array}$ & $u$ & $b$ & $v$ \\
\hline 6006.2 & $12.20 \pm 0.22$ & $12.09 \pm 0.32$ & $12.16 \pm 0.12$ & $12.32 \pm 0.08$ & $13.56 \pm 0.08$ & $13.71 \pm 0.07$ \\
\hline 6008.0 & $12.83 \pm 0.29$ & $\cdots$ & $\ldots$ & $\cdots$ & $\ldots$ & $\ldots$ \\
\hline 6009.8 & $\ldots$ & $\ldots$ & $12.64 \pm 0.09$ & $\ldots$ & $\ldots$ & $\ldots$ \\
\hline 6009.9 & $13.34 \pm 0.22$ & $\cdots$ & $\ldots$ & $\ldots$ & $\cdots$ & $\cdots$ \\
\hline 6011.9 & $\ldots$ & $\ldots$ & 12.56 & $\cdots$ & $\cdots$ & $\ldots$ \\
\hline 6012.2 & $13.77 \pm 0.22$ & $\ldots$ & $\ldots$ & $\ldots$ & $\ldots$ & $\ldots$ \\
\hline 6012.5 & $\ldots$ & $\ldots$ & $\ldots$ & $12.47 \pm 0.14$ & $\ldots$ & $\ldots$ \\
\hline 6012.8 & $\ldots$ & $\ldots$ & $12.90 \pm 0.12$ & $\ldots$ & $\ldots$ & $\cdots$ \\
\hline 6014.0 & $14.16 \pm 0.25$ & $14.01 \pm 0.21$ & $13.17 \pm 0.21$ & $12.52 \pm 0.12$ & $13.53 \pm 0.11$ & $13.50 \pm 0.10$ \\
\hline 6015.2 & $\ldots$ & $14.41 \pm 0.20$ & $\ldots$ & $\ldots$ & $\ldots$ & $\ldots$ \\
\hline 6015.5 & $14.52 \pm 0.11$ & $\ldots$ & $\ldots$ & $\ldots$ & $\ldots$ & $\ldots$ \\
\hline 6015.9 & $14.63 \pm 0.21$ & $14.64 \pm 0.09$ & $13.56 \pm 0.19$ & $12.60 \pm 0.12$ & $13.55 \pm 0.11$ & $13.53 \pm 0.10$ \\
\hline 6017.0 & $14.91 \pm 0.17$ & $\ldots$ & $\ldots$ & $12.71 \pm 0.27$ & $\ldots$ & $\ldots$ \\
\hline 6018.0 & $15.18 \pm 0.16$ & $15.30 \pm 0.12$ & $14.04 \pm 0.14$ & $12.80 \pm 0.12$ & $13.58 \pm 0.10$ & $13.50 \pm 0.09$ \\
\hline 6018.6 & $15.39 \pm 0.10$ & $15.68 \pm 0.08$ & $\ldots$ & $\ldots$ & $\ldots$ & $\ldots$ \\
\hline 6019.9 & $15.87 \pm 0.14$ & $16.10 \pm 0.10$ & $14.55 \pm 0.14$ & $13.04 \pm 0.14$ & $13.65 \pm 0.11$ & $13.46 \pm 0.11$ \\
\hline 6024.0 & $17.00 \pm 0.11$ & $17.64 \pm 0.09$ & $15.53 \pm 0.11$ & $13.76 \pm 0.14$ & $13.79 \pm 0.13$ & $13.45 \pm 0.11$ \\
\hline 6025.9 & $17.29 \pm 0.11$ & $18.17 \pm 0.10$ & $15.93 \pm 0.11$ & $14.08 \pm 0.14$ & $13.86 \pm 0.14$ & $13.45 \pm 0.13$ \\
\hline 6028.1 & $17.50 \pm 0.11$ & $18.36 \pm 0.10$ & $16.10 \pm 0.09$ & $14.39 \pm 0.12$ & $13.96 \pm 0.12$ & $13.46 \pm 0.11$ \\
\hline 6029.0 & $17.56 \pm 0.10$ & $\ldots$ & $\ldots$ & $14.56 \pm 0.31$ & $\ldots$ & $\cdots$ \\
\hline 6029.6 & $17.61 \pm 0.10$ & $\ldots$ & $\ldots$ & $\ldots$ & $\ldots$ & $\ldots$ \\
\hline 6029.7 & $17.72 \pm 0.12$ & $\ldots$ & $\ldots$ & $\ldots$ & $\ldots$ & $\ldots$ \\
\hline 6030.7 & $17.72 \pm 0.10$ & $18.94 \pm 0.13$ & $16.53 \pm 0.08$ & $14.77 \pm 0.10$ & $14.09 \pm 0.11$ & $13.51 \pm 0.10$ \\
\hline 6031.9 & $17.78 \pm 0.10$ & $\ldots$ & $16.53 \pm 0.09$ & $\ldots$ & $\ldots$ & $\ldots$ \\
\hline 6032.7 & $17.86 \pm 0.13$ & $\ldots$ & $\ldots$ & $14.82 \pm 0.09$ & $\ldots$ & $\cdots$ \\
\hline 6033.8 & $17.98 \pm 0.11$ & $19.33 \pm 0.16$ & $16.65 \pm 0.08$ & $14.97 \pm 0.09$ & $14.19 \pm 0.10$ & $13.54 \pm 0.09$ \\
\hline 6035.8 & $18.11 \pm 0.11$ & $19.44 \pm 0.15$ & $16.87 \pm 0.09$ & $15.20 \pm 0.11$ & $14.27 \pm 0.13$ & $13.60 \pm 0.12$ \\
\hline 6039.6 & $\ldots$ & $19.52 \pm 0.16$ & $\ldots$ & $\ldots$ & $\ldots$ & $13.61 \pm 0.10$ \\
\hline 6039.7 & $18.29 \pm 0.11$ & $\cdots$ & $16.95 \pm 0.09$ & $15.45 \pm 0.10$ & $14.36 \pm 0.13$ & $\cdots$ \\
\hline 6043.7 & $18.48 \pm 0.12$ & $19.62 \pm 0.19$ & $17.09 \pm 0.09$ & $15.72 \pm 0.08$ & $14.46 \pm 0.10$ & $13.62 \pm 0.09$ \\
\hline 6045.7 & $18.42 \pm 0.11$ & $19.66 \pm 0.17$ & $17.19 \pm 0.09$ & $15.76 \pm 0.09$ & $14.52 \pm 0.12$ & $13.64 \pm 0.11$ \\
\hline 6046.7 & $18.50 \pm 0.11$ & $19.91 \pm 0.20$ & $17.28 \pm 0.09$ & $15.90 \pm 0.09$ & $14.54 \pm 0.13$ & $13.64 \pm 0.11$ \\
\hline 6050.9 & $18.63 \pm 0.12$ & $20.19 \pm 0.23$ & $17.37 \pm 0.09$ & $16.14 \pm 0.09$ & $14.64 \pm 0.13$ & $13.66 \pm 0.12$ \\
\hline 6052.0 & $18.60 \pm 0.12$ & $19.83 \pm 0.22$ & $17.38 \pm 0.09$ & $16.12 \pm 0.08$ & $14.69 \pm 0.10$ & $13.70 \pm 0.09$ \\
\hline 6054.2 & $18.47 \pm 0.11$ & $20.08 \pm 0.22$ & $17.51 \pm 0.09$ & $16.20 \pm 0.09$ & $14.67 \pm 0.12$ & $13.64 \pm 0.11$ \\
\hline 6057.8 & $18.65 \pm 0.12$ & $20.40 \pm 0.28$ & $17.61 \pm 0.09$ & $16.40 \pm 0.08$ & $14.80 \pm 0.11$ & $13.68 \pm 0.11$ \\
\hline 6061.8 & $18.82 \pm 0.12$ & $20.53 \pm 0.32$ & $17.68 \pm 0.09$ & $16.63 \pm 0.08$ & $14.84 \pm 0.11$ & $13.73 \pm 0.10$ \\
\hline 6066.3 & $\ldots$ & $\ldots$ & $17.88 \pm 0.16$ & $\cdots$ & $\ldots$ & $\ldots$ \\
\hline 6074.1 & $19.30 \pm 0.16$ & $20.26 \pm 0.29$ & $17.82 \pm 0.10$ & $17.02 \pm 0.09$ & $15.03 \pm 0.09$ & $13.79 \pm 0.09$ \\
\hline 6078.2 & $18.92 \pm 0.13$ & $20.33 \pm 0.29$ & $17.92 \pm 0.10$ & $17.07 \pm 0.09$ & $15.11 \pm 0.10$ & $13.83 \pm 0.09$ \\
\hline 6113.4 & $19.67 \pm 0.20$ & 20.70 & $18.42 \pm 0.13$ & $18.11 \pm 0.15$ & $15.66 \pm 0.08$ & $14.22 \pm 0.08$ \\
\hline $\begin{array}{l}\text { UV GRISM } \\
\text { Number }\end{array}$ & $\begin{array}{l}\text { Start Time } \\
\text { (UT) }\end{array}$ & $\begin{array}{l}\text { Stop Time } \\
\text { (UT) }\end{array}$ & $\begin{array}{l}\text { Start Time } \\
\text { (JD) }\end{array}$ & $\begin{array}{l}\text { Stop Time } \\
\text { (JD) }\end{array}$ & $\begin{array}{l}\text { Exposure } \\
\text { (s) }\end{array}$ & \\
\hline 1 & 2012-03-21T00:16:24 & 2012-03-21T23:03:24 & 2456007.51 & 2456008.46 & 8287.4 & \\
\hline 2 & 2012-03-23Т00:41:04 & 2012-03-24T21:42:08 & 2456009.52 & 2456011.40 & 5116.7 & \\
\hline 3 & 2012-03-25Т00:48:24 & 2012-03-26T01:09:46 & 2456011.53 & 2456012.55 & 8021.7 & \\
\hline 4 & 2012-03-28T08:47:25 & 2012-03-28T23:43:28 & 2456014.87 & 2456015.49 & 5663.9 & \\
\hline 5 & 2012-03-30Т05:44:24 & 2012-03-30T18:43:24 & 2456016.74 & 2456017.28 & 8978.1 & \\
\hline 6 & 2012-04-11T01:42:00 & 2012-04-11T19:45:40 & 2456028.57 & 2456032.72 & 22759.8 & \\
\hline Number & $u v w 2^{\mathrm{b}}$ & $\begin{array}{l}\text { Spectrophotometric } \\
\text { uvm } 2\end{array}$ & $\begin{array}{c}\text { Magnitudes } \\
u v w 1\end{array}$ & $u$ & $b$ & $v^{\mathrm{b}}$ \\
\hline 1 & 12.979 & 12.494 & 12.348 & 12.327 & 13.335 & 13.019 \\
\hline 2 & 13.467 & 12.963 & 12.545 & 12.344 & 13.353 & 13.028 \\
\hline 3 & 14.701 & 13.522 & 12.897 & 12.390 & 13.330 & 12.989 \\
\hline 4 & 14.867 & 14.520 & 13.566 & 12.560 & 13.369 & 13.063 \\
\hline 5 & 15.050 & 14.929 & 13.934 & 12.685 & 13.435 & 13.120 \\
\hline 6 & $-^{\mathrm{c}}$ & $-^{\mathrm{c}}$ & $-^{\mathrm{c}}$ & 14.649 & 14.059 & 13.482 \\
\hline
\end{tabular}

Notes.

${ }^{\text {a }}$ Photometry values in italics are $3 \sigma$ upper limits. All photometric errors are $3 \sigma$.

b The spectrophotometric magnitudes for $u v w 2$ and $v$ are only limits as the UV grism does not fill these bandpasses.

c The last spectrum blueward of $2940 \AA$ is in the noise level. 


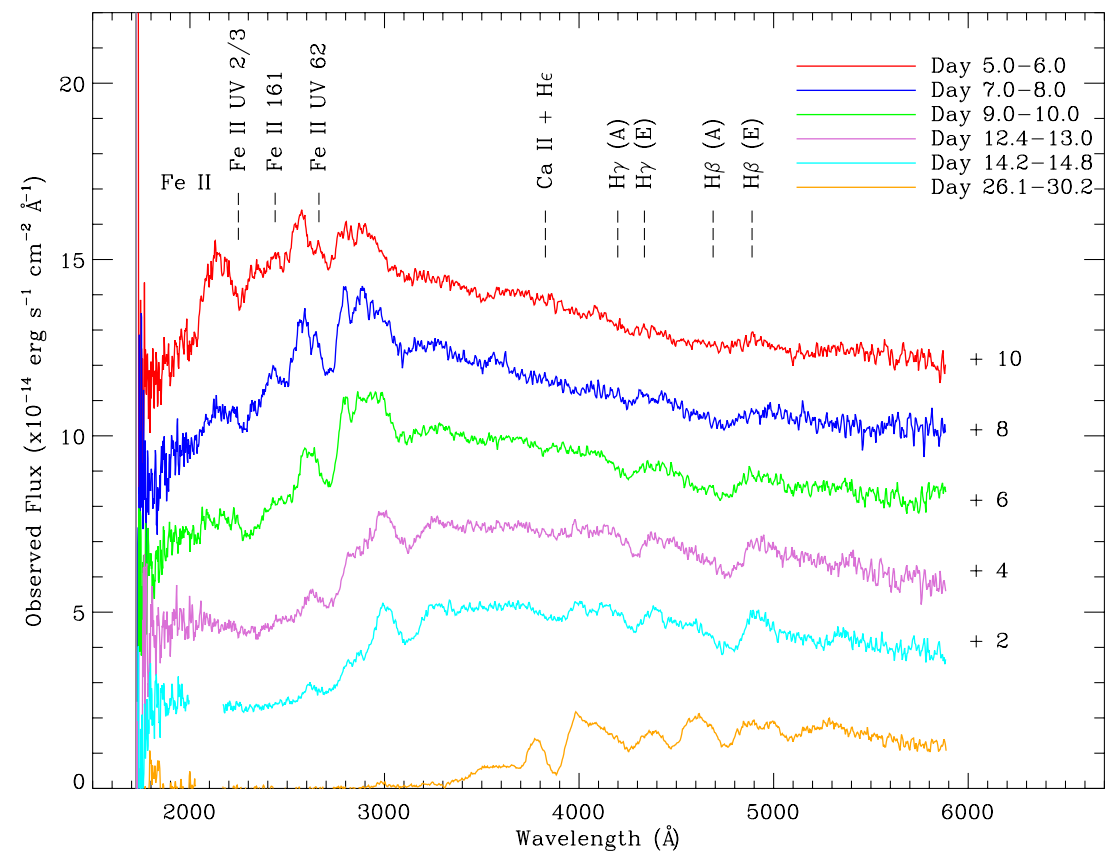

Figure 2. Time-series spectra from the UVOT UV grism. The spectra are offset from each other by adding a constant amount of flux for clarity. The individual hydrogen absorption (A) and emission (E) components are also labeled.

(A color version of this figure is available in the online journal.)

of the noise level. This spectrophotometry is consistent with the photometry to within the errors on the spectra of $\sim 5 \%-10 \%$. Because of this and that the UVOT photometry is will studied, we are confident that the UV plateau is a real feature of the SN 2012aw light curve.

\section{DISCUSSION}

\subsection{UV Light Curve}

The UV light curve fell rapidly and then flattened out approximately one month after the explosion. Unlike many UV filters used, $u v m 2$ has effectively no red leak and therefore we can be confident that the UV plateau is a real feature of the $\mathrm{SN}$. There is a red leak in $u v w 1$ and $u v w 2$, but applying red leak correction (Brown et al. 2010) still shows the plateau, just $\sim 2.5$ mag fainter in both filters. The red leak does not contribute at all to the early-time light curve up to $\sim$ Day 21 .

Figure 3 shows the UV and $u$-band light curve of SN 2012aw along with other Type II-P SNe corrected for distance, but not extinction since the extinction in the host galaxy for several of these is unknown. The worst case scenario is likely SN 2006bc, which has a total $E(B-V)=0.52$, corresponding to $\sim 4.3 \mathrm{mag}$ change in $u v w 2$ and $u v m 2, \sim 3.5 \mathrm{mag}$ change in $u v w 1$, and $\sim 2.6$ mag change in $u$ band, assuming the extinction of Cardelli et al. (1989). This flattening to the UV slope at late times is suggested, but not well observed in the prior UV studies of Type II-P SNe, as the UV flux fell below detection levels within a few weeks. We also show the ground-based $U$ band for SN 1999em and 2004dj, which do extend past 100 days. Both of these also show a flat $U$-band slope, like SN 2012aw. SN 2012aw's UV light curve appears similar in shape to that of SN 2006at, SN 2006bp, and possibly SN 2006bc, although the latter has very few observations. The UV light curves of SN 2005cs also appear similar if the light curves are shifted forward by a few days.

\subsection{UV Spectral Evolution}

In Figure 2, the Swift UV spectra are plotted. Since the UVOT is a photon-counting instrument, the flux errors are well understood. They are smallest around $3000 \AA$, where the sensitivity is highest, and largest below $1800 \AA$ and above $4000 \AA$. The brightness of the SN is low enough that effects of coincidence loss (Poole et al. 2008; Kuin \& Rosen 2008; P. Kuin et al., in preparation) are small and dominated by the coincidence loss in the background (Breeveld et al. 2010). These UV spectra are the most detailed UV spectra of an early Type II-P SN. The final spectrum taken two weeks after explosion still shows some near-UV emission.

In Figure 2, the main spectral features are identified. The main spectral absorption minima are blueshifted by $6000-8000 \mathrm{~km} \mathrm{~s}^{-1}$, while the edge velocities in $\mathrm{H} \beta$ and $\mathrm{H} \gamma$ P-Cygni profiles are $\sim 15,000$ and $\sim 13,000 \mathrm{~km} \mathrm{~s}^{-1}$. The UV end of the spectrum decreases in brightness rapidly over the course of the month. This decrease is likely due to iron line blanketing with the addition of nickel after $\sim 10$ days

Figure 4 shows the SN 2012aw observed spectra along with two spectral models for nearly the same post-explosion time. The models are from Dessart et al. (2008) and were originally created from the analysis of Swift UVOT data of SN $2005 \mathrm{cs}$ and SN 2006bp. The model parameters of SN 2006bp and 2005cs (given in Table 6 and Table 7 of Dessart et al. 2008) have been extinction corrected from the empirical extinction formula of Cardelli et al. (1989) using the same Milky Way reddening for SN 2012aw of $E(B-V)=0.025$ mag (Schlafly \& Finkbeiner 2011). Van Dyk et al. (2012) have shown that the reddening of the host galaxy is $E(B-V)=0.08$, but Kochanek et al. (2012) suggest that it is even less.

Gal-Yam et al. (2008) suggest that all Type II-P SNe should be very similar, which is seen here with the SN 2012aw spectrum falling largely in between the two models. According to Dessart et al. (2008), the main difference between SN 2006bp and 


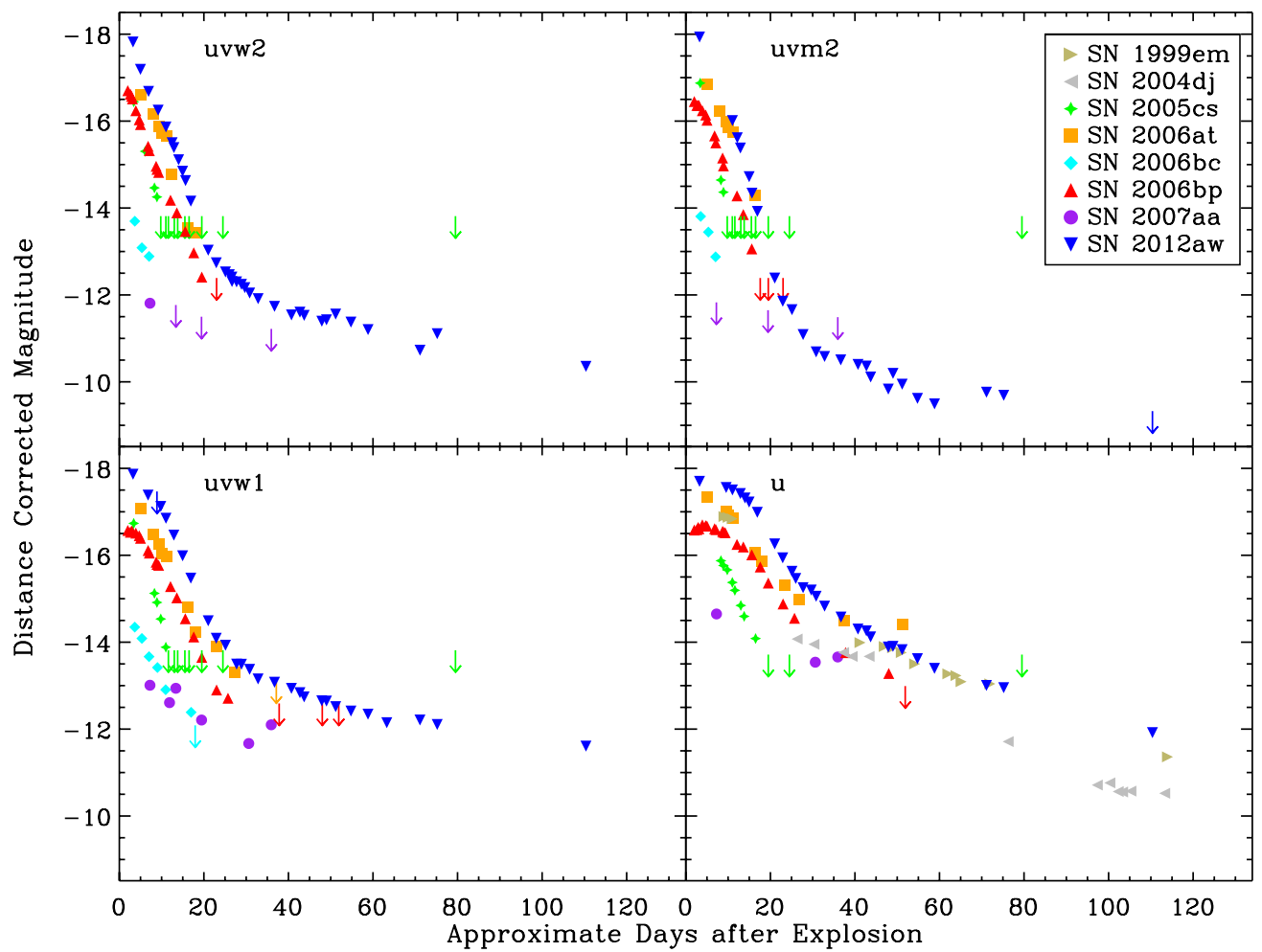

Figure 3. Type II-P SNe UV and $u$-band light curves corrected for distance, but not extinction. The light curves from SN 1999em are from Elmhamdi et al. (2003), SN 2004dj from Tsvetkov et al. (2008), SN 2005cs and SN 2006bp from Dessart et al. (2008), and SN 2006at, SN 2006bc, and SN 2007aa from Brown et al. (2009). The arrows indicate upper limits.

(A color version of this figure is available in the online journal.)

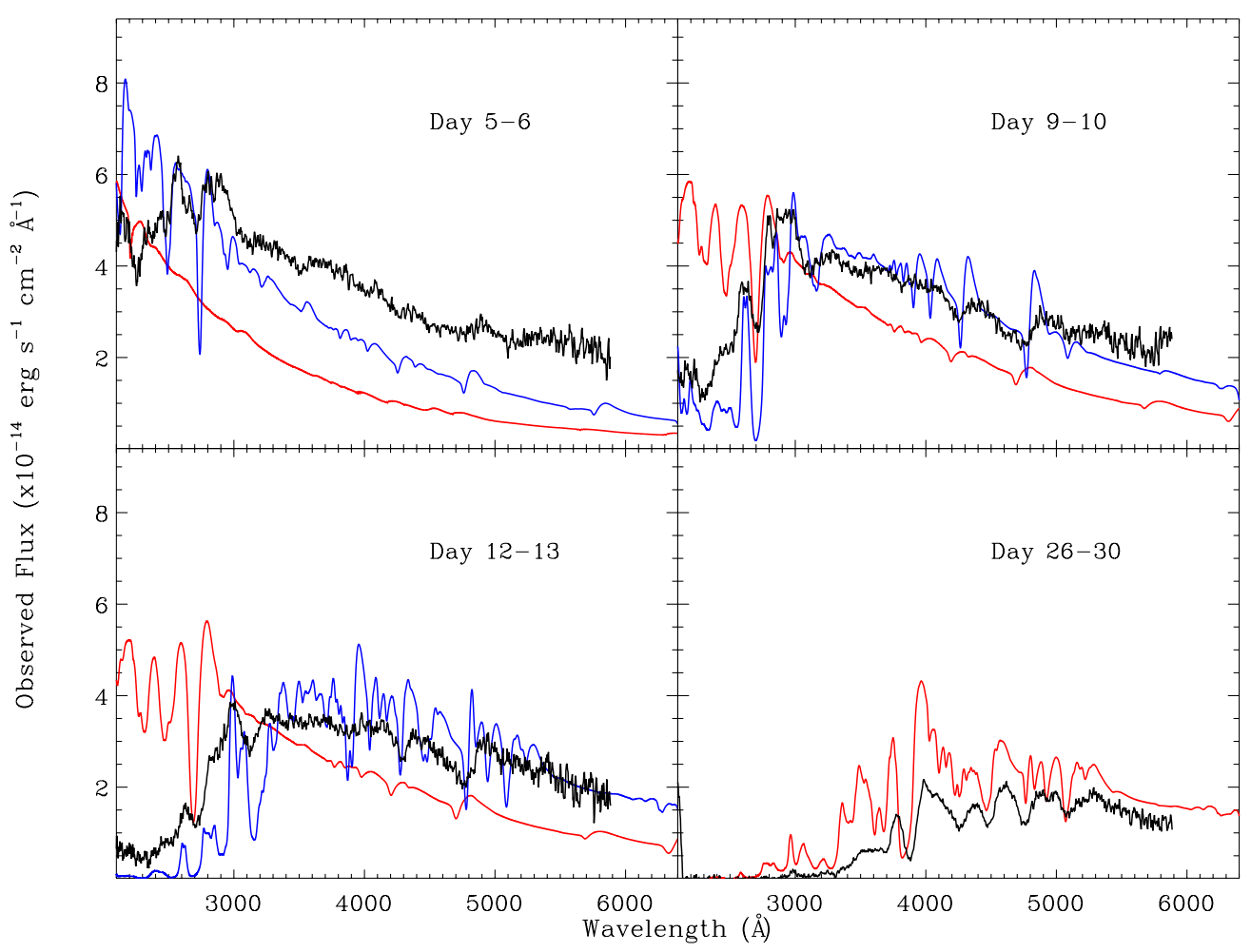

Figure 4. UV time-series spectrum of SN 2012aw (black) with the spectral models (Dessart et al. 2008) for SN 2005cs (blue) and SN 2006bp (red) scaled by eye to the SN 2012aw observations and extinction applied using the reddening to SN 2012aw.

(A color version of this figure is available in the online journal.) 
SN 2005cs is that SN 2005cs may be intrinsically faint with low kinetic energy in the ejecta. However, it is not surprising that there are some discrepancies between the models and the observations of SN 2012aw. The best UV spectral models are based on early-time spectra and late-time photometry, including these. Now that there are late-time UV spectral observations of a normal Type II-P (versus the peculiar SN 1987A); improvements to the spectral models can be made.

\section{CONCLUSIONS}

SN 2012aw shows the same optical light curve behavior as other Type II-P SNe, namely an optical plateau due to the reprocessing of hydrogen recombination emission. This $\mathrm{SN}$ was also UV bright early on and faded rapidly in the same manner as other observed Type II-P SNe. However, at late epochs, after Day 27, the light curve slope flattened to a much slower decline. At these later times, the UV light curve uniquely gives information on temperature changes. Thus, the UV flattening indicates that the temperature is remaining constant, which requires a source of heating to maintain the photospheric temperature. Some possible sources of late-time heating are continued recombination, radioactive decay, or a combination of these.

Past Day 27, as the UV slope flattens, the $u v m 2-v$ color flattens and the temperature flattens. The bolometric temperature as calculated from the UVOT filters $u v w 1$ and $u v w 2$ and groundbased UBVRIJHK (M. Dall'Ora et al., in preparation) becomes constant at $\sim 4500 \mathrm{~K}$. A blackbody temperature of $4500 \mathrm{~K}$ would have a color of $u v m 2-v=4.1 \mathrm{mag}$ including the Van Dyk et al. (2012) reddening, but we observe $u v m 2-v \sim 6$ mag. The difference in color is likely due to line blanketing. We note, however, that there have been some indications of a higher reddening value from Dall'Ora et al. (in preparation) if there is significant dust present after the explosion. They suggest an $E(B-V)=0.27$, which would produce a $4500 \mathrm{~K}$ blackbody color of $u v m 2-v=5.3$. If this is so, then line blanketing may be less significant and the $\mathrm{SN}$ was intrinsically brighter.

The UV plateau in SN 2012aw is not seen in the other UV-observed SNe because most were either too extincted (SN 2006bc: $E(B-V)=0.19$-line of sight, Schlafly \& Finkbeiner 2011; $E(B-V)=0.33$-host, Otsuka et al. 2012) or too far away (SN 2007aa: 20.5 Mpc, Smartt et al. 2009; SN 2006at: $64 \mathrm{Mpc}$, Blondin et al. 2006). SN 2005cs was nearby (in M51) and not very extincted, but Dessart et al. (2008) suggest that it was sub-luminous. SN 2006bp is the best comparison and has indications that the light curve was beginning to flatten, but it too was fairly distant and extincted $(17.5 \mathrm{Mpc}, E(B-V)=0.37$ host; Dessart et al. 2008) and was not detected in the UV approximately a month after explosion. Thus, we have lengthy observations of a normal Type II-P SN that is nearby allowing us to observe the UV plateau for the first time.
Because of SN 2012aw seeming similarity to SN 2006bp and at least the initial data of the other SNe, this UV plateau phase could be ubiquitous. ${ }^{15}$ However, as SN 2012aw is the first we can only confirm this with additional observations of nearby Type II-P SNe.

The authors thank Luc Dessart for providing the digital models for SN 2005cs and SN 2006bp. This research has made use of the NASA/IPAC Extragalactic Database (NED) which is operated by the Jet Propulsion Laboratory, California Institute of Technology, under contract with the National Aeronautics and Space Administration. The authors thank the anonymous referee for insightful comments.

\section{REFERENCES}

Baron, E., Branch, D., Hauschildt, P. H., et al. 2000, ApJ, 545, 444 Blondin, S., Modjaz, M., Kirshner, R., et al. 2006, CBET, 441, 1 Breeveld, A. A., Landsman, W., Holland, S. T., et al. 2011, in AIP Conf. Proc. 1358, Gamma Ray Bursts 2010, ed. J. E. McEnery, J. L. Racusin, \& N. Gehrels (Melville, NY: AIP), 373

Breeveld, A. A., Curran, P. A., Hoversten, E. A., et al. 2010, MNRAS, 406, 1687

Brown, P. J., Dessart, L., Holland, S. T., et al. 2007, ApJ, 659, 1488

Brown, P. J., Holland, S. T., Immler, S., et al. 2009, AJ, 137, 4517

Brown, P. J., Roming, P. W. A., Milne, P., et al. 2010, ApJ, 721, 1608

Cardelli, J. A., Clayton, G. C., \& Mathis, J. S. 1989, ApJ, 345, 245

Cassatella, A., Fransson, C., vant Santvoort, J., et al. 1987, A\&A, 177, L29

Dessart, L., Blondin, S., Brown, P. J., et al. 2008, ApJ, 675, 644

Elmhamdi, A., Danziger, I. J., Chugai, N., et al. 2003, MNRAS, 338, 939

Fagotti, P. 2012, CBET, 3054, 1

Fraser, M., Maund, J. R., Smartt, S. J., et al. 2012, ApJL, 759, L13

Freedman, W. L., Madore, B. F., Gibson, B. K., et al. 2001, ApJ, 553, 47

Gal-Yam, A., Bufano, F., Barlow, T. A., et al. 2008, ApJL, 685, L117

Gehrels, N., Chincarini, G., Giommi, P., et al. 2004, ApJ, 611, 1005

Itoh, R., Ui, T., \& Yamanaka, M. 2012, CBET, 3054, 2

Kirshner, R. P., \& Kwan, J. 1975, ApJ, 197, 415

Kirshner, R. P., Oke, J. B., Penston, M. V., \& Searle, L. 1973, ApJ, 185, 303

Kochanek, C. S., Khan, R., \& Dai, X. 2012, ApJ, 759, 20

Kuin, N. P. M., \& Rosen, S. R. 2008, MNRAS, 383, 383

Leonard, D. C., Filippenko, A. V., Li, W., et al. 2002, AJ, 124, 2490

Li, W., Leaman, J., Chornock, R., et al. 2011, MNRAS, 412, 1441

Mitchell, R. C., Baron, E., Branch, D., et al. 2002, ApJ, 574, 293

Munari, U. 2012, CBET, 3054, 3

Otsuka, M., Meixner, M., Panagia, N., et al. 2012, ApJ, 744, 26

Poole, T. S., Breeveld, A. A., Page, M. J., et al. 2008, MNRAS, 383, 627

Poznanski, D., Nugent, P. E., Ofek, E. O., Gal-Yam, A., \& Kasliwal, M. M. 2012, ATel, 3996, 1

Pun, C. S. J., Kirshner, R. P., Sonneborn, G., et al. 1995, ApJS, 99, 223

Roming, P. W., Townsley, L. K., Nousek, J. A., et al. 2000, Proc. SPIE, 4140, 76

Roming, P. W. A., Hunsberger, S. D., Mason, K. O., et al. 2004, Proc. SPIE, 5165,262

Roming, P. W. A., Kennedy, T. E., Mason, K. O., et al. 2005, SSRv, 120, 95

Roming, P. W. A., Pritchard, T. A., Prieto, J. L., et al. 2012, ApJ, 751, 92

Schlafly, E. F., \& Finkbeiner, D. P. 2011, ApJ, 737, 103

Siviero, A., Tomasella, L., Pastorello, A., Benetti, S., \& Munari, U. 2012, CBET, 3054, 4

Smartt, S. J., Eldridge, J. J., Crockett, R. M., \& Maund, J. R. 2009, MNRAS, 395, 1409

Tsvetkov, D. Y., Goranskij, V., \& Pavlyuk, N. 2008, PZ, 28, 8

Van Dyk, S. D., Cenko, S. B., Poznanski, D., et al. 2012, ApJ, 756, 131
15 We note that galactic template subtractions are sometimes done with post-explosion data. We caution that there may be SN UV flux even at late times in low-extinction SNe. 